\title{
Brønsted acidic ionic liquid catalysis: An efficient and eco-friendly synthesis of novel fused pyrano pyrimidinones and their antimicrobial activity
}

\author{
JANARDHAN BANOTHU, RAJITHA GALI, RAVIBABU VELPULA \\ and RAJITHA BAVANTULA* \\ Department of Chemistry, National Institute of Technology, Warangal 506 004, India \\ e-mail: rajitha_nitw@yahoo.com; rajitabhargavi@yahoo.com
}

MS received 30 October 2012; revised 11 February 2013; accepted 5 April 2013

\begin{abstract}
A series of novel fused pyrano pyrimidinones were synthesized by the condensation of 2-amino4-aryl-4H-benzo[h]chromene-3-carbonitriles with 2-oxo- $2 \mathrm{H}$-chromene-3-carboxylic acid under neat conditions employing an efficient, eco-friendly and reusable Brønsted acidic ionic liquid, (4-sulfobutyl)tris(4sulfophenyl)phosphonium hydrogen sulphate as catalyst. All the synthesized compounds were characterized by their analytical and spectroscopic data and they were screened for in vitro antibacterial activity against Bacillus subtilis, Staphylococcus aureus and Staphylococcus epidermidis as Gram- positive, Escherichia coli, Pseudomonas aeruginosa and Klebsiella pneumonia as Gram-negative bacterial strains and antifungal activity against Aspergillus flavus, Saccharomyces cerevisiae, Candida rugosa and Candida albicans. Compounds 2c and $\mathbf{2 d}$ have shown good antibacterial activity against Escherichia coli while $\mathbf{2 g}$ has shown marked antifungal activity against Candida rugosa.
\end{abstract}

Keywords. Antibacterial activity; antifungal activity; Brønsted acidic ionic liquid; fused pyrano pyrimidinones; neat conditions.

\section{Introduction}

Recent studies reveal that bacterial infections have increased at an alarming rate causing deadly diseases and wide spread epidemics in humans. Pyran derivatives possess a wide range of medicinal applications such as antimicrobial, ${ }^{1-4}$ antitubercular, ${ }^{4}$ antioxidant, ${ }^{5}$ antigenotoxic, ${ }^{6}$ cytotoxic, ${ }^{7}$ ATP sensitive potassium channel openers ${ }^{8}$ and antiangiogenic activities. ${ }^{9}$ On the other hand, coumarins and pyrimidines were also found to possess several biological activities like antimicrobial, ${ }^{10-14}$ antiviral, ${ }^{15}$ anticonvulsant, ${ }^{16}$ anticoagulant, ${ }^{17}$ antioxidant, ${ }^{18}$ anti-HIV, ${ }^{19}$ antihistamine,${ }^{20}$ antitubercular, ${ }^{21}$ antitumour, ${ }^{22}$ antiinflammatory ${ }^{23,24}$ and antileishmanial activities. ${ }^{25}$ Several methods have been reported for the synthesis of pyrimidinones using $\mathrm{Yb}(\mathrm{OTf})_{3}$ in ionic liquids, ${ }^{26} \mathrm{Zn}(\mathrm{OAc})_{2}$ under microwave irradiation, ${ }^{27}$ cyanuric chloride ${ }^{28}$ and acidic ionic liquid ${ }^{29}$ as catalysts. Most of these reported methods are involving multistep process and suffering from one or several drawbacks such as low yield, long reaction time, tedious work-up, harsh reaction conditions and use of large quantity of expensive reagents. Moreover,

*For correspondence nowadays organic solvents were replaced by ionic liquids because of their interesting properties like high thermal stability, non-volatility, eco-friendly, high selectivity, high atom efficiency, easy separation from the reaction mixture and reusability. ${ }^{30}$ Similarly, reactions under solvent-free conditions ${ }^{31}$ usually need shorter reaction times and simple work-up procedures.

In view of the high degree of bio-activity shown by pyrans, coumarins and pyrimidines, we have focused on the design of a novel structural entity that incorporates these three structural moieties into a single molecular scaffold to evaluate the potential additive effect on antimicrobial activity. Also, in continuation of our earlier studies on pyrimidinones, ${ }^{32}$ we now elicit the synthesis of fused pyrano pyrimidinones utilizing inexpensive, eco-friendly and reusable Brønsted acidic ionic liquid as a catalyst under solvent-free conditions as marked antimicrobial agents.

\section{Experimental}

\subsection{Materials}

All commercially available reagents and solvents were employed without further purification. Melting points 
were determined in open capillaries using Stuart SMP30 apparatus and are uncorrected. The progress of the reactions as well as purity of compounds was monitored by thin layer chromatography with $\mathrm{F}_{254}$ silicagel pre-coated sheets (Merck, Darmstadt, Germany) using hexane/ethyl acetate $8 / 2$ as eluent; UV light was used for detection. IR spectra were recorded on PerkinElmer 237 spectrophotometer using $\mathrm{KBr}$ pellet, values are expressed in $\mathrm{cm}^{-1}$. NMR spectra were recorded on Bruker $300 \mathrm{MHz}$ spectrometer using DMSO as solvent and TMS as internal standard, chemical shifts are expressed in ppm. Elemental analysis was performed on a Carlo Erba modal EA1 108 and the values are $\pm 0.4 \%$ of the theoretical ones. Mass spectra were recorded on a Jeol JMSD-300 spectrometer.

\subsection{General procedure for the synthesis of fused pyrano pyrimidinones $(\mathbf{2 a}-\mathbf{j})$}

To a mixture of 2-amino-4-aryl-4H-benzo[ $h]$ chromene3-carbonitrile (1a-j, $1 \mathrm{mmol})$ and 2-oxo- $2 H$-chromene3 -carboxylic acid (1 mmol); ionic liquid $(10 \mathrm{~mol} \%)$ was added and heated at $120^{\circ} \mathrm{C}$ for about $8-12 \mathrm{~h}$ without any solvent. After completion of the reaction shown by TLC, $2 \mathrm{~mL}$ of water was added and stirred at RT for additional $5 \mathrm{~min}$. The solid separated out was filtered and purified over column chromatography using silica gel (230-400 mesh) with $n$-hexane and ethyl acetate $(8: 2)$ as eluent. The aqueous layer containing catalyst was recovered, washed with acetone, dried and reused for subsequent reactions without loss in its activity and product yield.

\subsection{Characterization of compounds $(2 a-j)$}

2.3a 10-(2-Oxo-2H-chromen-3-yl)-7-phenyl-9Hnaphtho[3', 4':5,6]pyrano[2,3-d]pyrimidin-8-one (2a): Pale yellow solid; IR (KBr) $v_{\max }\left(\mathrm{cm}^{-1}\right): 3289(\mathrm{NH})$, 1772 (CO of lactone), 1723 (CO of lactam), 1599 $(\mathrm{C}=\mathrm{N}), 1160(\mathrm{C}-\mathrm{O}-\mathrm{C}) ;{ }^{1} \mathrm{H}$ NMR $(300 \mathrm{MHz}$, DMSO$\left.d_{6}\right): \delta 5.29(\mathrm{~s}, 1 \mathrm{H}), 7.15-7.26(\mathrm{~m}, 5 \mathrm{H}), 7.34(\mathrm{~d}, J=$ $6.9 \mathrm{~Hz}, 1 \mathrm{H}), 7.41-7.45(\mathrm{~m}, 4 \mathrm{H}), 7.71-7.75(\mathrm{~m}, 1 \mathrm{H})$, $7.83(\mathrm{~d}, J=6.0 \mathrm{~Hz}, 1 \mathrm{H}), 7.90-7.93(\mathrm{~m}, 3 \mathrm{H}), 8.75(\mathrm{~s}$, $1 \mathrm{H}), 10.82(\mathrm{~s}, 1 \mathrm{H}) ;{ }^{13} \mathrm{CNMR}\left(75 \mathrm{MHz}, \mathrm{DMSO}-d_{6}\right)$ : 173.6, 164.8, 163.9, 159.6, 154.4, 146.8, 145.6, 134.2, $130.7,130.1,129.4,128.6,128.4,127.0,126.9,126.5$, 124.8, 124.7, 123.5, 120.4, 118.3, 117.9, 116.7, 116.0, 106.5, 38.0; MS: $m / z=509(\mathrm{M}+\mathrm{K})$; Anal. Calcd. For $\mathrm{C}_{30} \mathrm{H}_{18} \mathrm{~N}_{2} \mathrm{O}_{4}$ : C, 76.59; H, 3.86; N, 5.95; Found: C, 76.64; H, 3.72; N, 5.98.
$2.3 \mathrm{~b}$ 7-(2-Chlorophenyl)-10-(2-oxo-2H-chromen-3-yl)9H-naphtho[ $\left.3^{\prime}, 4^{\prime}: 5,6\right]$ pyrano[2,3-d]pyrimidin-8-one (2b): Colourless solid; IR $(\mathrm{KBr}) v_{\max }\left(\mathrm{cm}^{-1}\right): 3275$ $(\mathrm{NH}), 1768$ (CO of lactone), 1715 (CO of lactam), $1585(\mathrm{C}=\mathrm{N}), 1172(\mathrm{C}-\mathrm{O}-\mathrm{C}), 762(\mathrm{C}-\mathrm{Cl}) ;{ }^{1} \mathrm{H}$ NMR $\left(300 \mathrm{MHz}, \mathrm{DMSO}-d_{6}\right): \delta 5.42(\mathrm{~s}, 1 \mathrm{H}), 7.01(\mathrm{~d}, J=$ $6.3 \mathrm{~Hz}, 1 \mathrm{H}), 7.22-7.46(\mathrm{~m}, 6 \mathrm{H}), 7.59-7.65(\mathrm{~m}, 3 \mathrm{H})$, $7.72(\mathrm{~d}, J=5.7 \mathrm{~Hz}, 1 \mathrm{H}), 7.88-7.92(\mathrm{~m}, 2 \mathrm{H}), 8.24$ $(\mathrm{d}, J=6.0 \mathrm{~Hz}, 1 \mathrm{H}), 8.75(\mathrm{~s}, 1 \mathrm{H}), 10.86(\mathrm{~s}, 1 \mathrm{H})$; ${ }^{13} \mathrm{C}$ NMR $\left(75 \mathrm{MHz}, \mathrm{DMSO}-d_{6}\right)$ : 173.0, 164.7, 163.9, $160.3,154.4,142.9,142.1,134.1,132.7,131.9,131.1$, $130.1,129.7,128.8,127.8,127.6,126.8,126.6,125.3$, 124.7, 124.0, 122.6, 120.7, 120.0, 118.3, 117.9, 105.9, 38.1; MS: $m / z=505(\mathrm{M}+1)$; Anal. Calcd. For $\mathrm{C}_{30} \mathrm{H}_{17} \mathrm{ClN}_{2} \mathrm{O}_{4}$ : C, 71.36; H, 3.39; N, 5.55; Found: C, 71.62; H, 3.17; N, 5.84 .

2.3c 7-(4-Chlorophenyl)-10-(2-oxo-2H-chromen-3-yl)9H-naphtho[3', 4':5,6]pyrano[2,3-d]pyrimidin-8-one (2c): Colourless solid; IR $(\mathrm{KBr}) v_{\max }\left(\mathrm{cm}^{-1}\right): 3251$ $(\mathrm{NH}), 1763$ (CO of lactone), 1718 (CO of lactam), $1598(\mathrm{C}=\mathrm{N}), 1184(\mathrm{C}-\mathrm{O}-\mathrm{C}), 752(\mathrm{C}-\mathrm{Cl}) ;{ }^{1} \mathrm{H}$ NMR $\left(300 \mathrm{MHz}, \mathrm{DMSO}-d_{6}\right): \delta 4.95(\mathrm{~s}, 1 \mathrm{H}), 7.10(\mathrm{~d}, J=$ $6.6 \mathrm{~Hz}, 1 \mathrm{H}), 7.28(\mathrm{~d}, J=6.3 \mathrm{~Hz}, 2 \mathrm{H}), 7.37-7.45(\mathrm{~m}$, $4 \mathrm{H}), 7.58-7.64(\mathrm{~m}, 2 \mathrm{H}), 7.71-7.92(\mathrm{~m}, 4 \mathrm{H}), 8.24(\mathrm{~d}$, $J=6.0 \mathrm{~Hz}, 1 \mathrm{H}), 8.75(\mathrm{~s}, 1 \mathrm{H}), 10.96(\mathrm{~s}, 1 \mathrm{H}) ;{ }^{13} \mathrm{C} \mathrm{NMR}$ $\left(75 \mathrm{MHz}, \mathrm{DMSO}-d_{6}\right): 172.9,164.8,163.9,160.1$, $154.4,144.5,142.7,134.1,132.7,131.5,130.1,129.5$, 128.6, 127.6, 126.8, 126.6, 126.0, 124.7, 123.9, 122.7, 120.6, 120.2, 118.3, 117.9, 117.3, 106.3, 38.0; MS: $m / z=505(\mathrm{M}+1)$; Anal. Calcd. For $\mathrm{C}_{30} \mathrm{H}_{17} \mathrm{ClN}_{2} \mathrm{O}_{4}$ : C, 71.36; H, 3.39; N, 5.55; Found: C, 71.58; H, 3.21; N, 5.73 .

2.3d 7-(3-Nitrophenyl)-10-(2-oxo-2H-chromen-3-yl)9H-naphtho[3',4':5,6]pyrano[2,3-d]pyrimidin-8-one (2d): Colourless solid; IR (KBr) $v_{\max }\left(\mathrm{cm}^{-1}\right): 3302$ $(\mathrm{NH}), 1758$ (CO of lactone), 1709 (CO of lactam), $1604(\mathrm{C}=\mathrm{N}), 1526\left(\mathrm{NO}_{2}\right), 1169(\mathrm{C}-\mathrm{O}-\mathrm{C}) ;{ }^{1} \mathrm{H}$ NMR $\left(300 \mathrm{MHz}, \mathrm{DMSO}-d_{6}\right): \delta 5.21(\mathrm{~s}, 1 \mathrm{H}), 7.15(\mathrm{~d}, J=$ $6.3 \mathrm{~Hz}, 1 \mathrm{H}), 7.32-7.45(\mathrm{~m}, 4 \mathrm{H}), 7.60-7.66(\mathrm{~m}, 2 \mathrm{H})$, $7.71-7.76(\mathrm{~m}, 2 \mathrm{H}), 7.91(\mathrm{~d}, J=6.0 \mathrm{~Hz}, 2 \mathrm{H}), 8.12(\mathrm{~d}$, $J=6.6 \mathrm{~Hz}, 2 \mathrm{H}), 8.26(\mathrm{~d}, J=6.0 \mathrm{~Hz}, 1 \mathrm{H}), 8.75(\mathrm{~s}$, $1 \mathrm{H}), 10.84(\mathrm{~s}, 1 \mathrm{H}) ;{ }^{13} \mathrm{C}$ NMR $\left(75 \mathrm{MHz}, \mathrm{DMSO}-d_{6}\right)$ : $172.9,164.3,163.9,160.0,154.4,147.9,147.8,142.9$, $134.5,134.2,132.8,130.4,130.1,127.6,126.9,126.7$, 125.8, 124.7, 124.2, 122.7, 122.1, 122.0, 120.7, 120.1, 118.3, 117.9, 116.7, 106.2, 40.5; MS: $m / z=516$ $(\mathrm{M}+1)$; Anal. Calcd. For $\mathrm{C}_{30} \mathrm{H}_{17} \mathrm{~N}_{3} \mathrm{O}_{6}$ : C, 69.90; $\mathrm{H}$, 3.32; N, 8.15. Found: C, 69.95; H, 3.23; N, 8.30. 
$2.3 \mathrm{e}$ 10-(2-Oxo-2H-chromen-3-yl)-7-p-tolyl-9H-naphtho$\left[3^{\prime}, 4^{\prime}: 5,6\right]$ pyrano[2,3-d]pyrimidin-8-one (2e): Pale yellow solid; IR (KBr) $v_{\max }\left(\mathrm{cm}^{-1}\right): 3298(\mathrm{NH}), 1752$ (CO of lactone), 1706 (CO of lactam), $1594(\mathrm{C}=\mathrm{N})$, $1178(\mathrm{C}-\mathrm{O}-\mathrm{C}) ;{ }^{1} \mathrm{H}$ NMR $\left(300 \mathrm{MHz}, \mathrm{DMSO}-d_{6}\right): \delta 2.25$ (s, 3H), $4.84(\mathrm{~s}, 1 \mathrm{H}), 7.08-7.12(\mathrm{~m}, 3 \mathrm{H}), 7.39-7.45(\mathrm{~m}$, $3 \mathrm{H}), 7.57-7.63(\mathrm{~m}, 3 \mathrm{H}), 7.71-7.92(\mathrm{~m}, 4 \mathrm{H}), 8.23(\mathrm{~d}$, $J=6.3 \mathrm{~Hz}, 1 \mathrm{H}), 8.75(\mathrm{~s}, 1 \mathrm{H}), 10.86(\mathrm{~s}, 1 \mathrm{H}) ;{ }^{13} \mathrm{C} \mathrm{NMR}$ $\left(75 \mathrm{MHz}, \mathrm{DMSO}-d_{6}\right)$ : $174.2,164.7,163.9,160.0$, 154.4, 142.7, 142.6, 136.0, 134.2, 132.6, 130.1, 129.2, $127.6,127.5,126.6,126.5,126.2,124.7,123.7,122.7$, 120.6, 120.4, 118.3, 118.0, 117.9, 106.4, 40.5, 20.5; MS: $m / z=485(\mathrm{M}+1)$; Anal. Calcd. For $\mathrm{C}_{31} \mathrm{H}_{20} \mathrm{~N}_{2} \mathrm{O}_{4}$ : C, 76.85; H, 4.16; N, 5.78. Found: C, 76.92; H, 4.01; N, 5.89.

$2.3 \mathrm{f}$ 7-(4-Methoxyphenyl)-10-(2-oxo-2H-chromen3-yl)-9H-naphtho[3', 4':5,6]pyrano[2,3-d]pyrimidin-8one (2f): Pale yellow solid; IR $(\mathrm{KBr}) v_{\max }\left(\mathrm{cm}^{-1}\right)$ : $3273(\mathrm{NH}), 1763$ (CO of lactone), 1721 (CO of lactam), $1608(\mathrm{C}=\mathrm{N}), 1161,1134(\mathrm{C}-\mathrm{O}-\mathrm{C}) ;{ }^{1} \mathrm{H}$ NMR $\left(300 \mathrm{MHz}, \mathrm{DMSO}-d_{6}\right): \delta 3.71(\mathrm{~s}, 3 \mathrm{H}), 4.84(\mathrm{~s}, 1 \mathrm{H})$, $6.88(\mathrm{~d}, J=6.6 \mathrm{~Hz}, 1 \mathrm{H}), 7.09(\mathrm{~d}, J=6.3 \mathrm{~Hz}, 2 \mathrm{H})$, $7.16(\mathrm{~d}, J=6.3 \mathrm{~Hz}, 2 \mathrm{H}), 7.39-7.45(\mathrm{~m}, 2 \mathrm{H}), 7.55-$ $7.65(\mathrm{~m}, 3 \mathrm{H}), 7.71-7.92(\mathrm{~m}, 3 \mathrm{H}), 8.24(\mathrm{~d}, J=6.0 \mathrm{~Hz}$, $1 \mathrm{H}), 8.75(\mathrm{~s}, 1 \mathrm{H}), 10.82(\mathrm{~s}, 1 \mathrm{H}) ;{ }^{13} \mathrm{C} \mathrm{NMR}(75 \mathrm{MHz}$, DMSO- $\left.d_{6}\right)$ : $173.0,164.6,163.8,160.0,157.8,154.3$, 144.2, 142.7, 132.9, 132.7, 129.5, 128.6, 127.6, 126.7, $126.5,126.1,124.9,123.9,122.7,120.6,120.3,118.5$, 117.9, 117.4, 115.7, 106.9, 55.4, 38.1; MS: $m / z=501$ $(\mathrm{M}+1)$; Anal. Calcd. For $\mathrm{C}_{31} \mathrm{H}_{20} \mathrm{~N}_{2} \mathrm{O}_{5}$ : C, 74.39; $\mathrm{H}$, 4.03; N, 5.60. Found: C, 74.62; H, 4.38; N, 5.27.

2.3g 7-(3,4-Dimethoxyphenyl)-10-(2-oxo-2H-chromen3-yl)-9H-naphtho[3', 4':5,6]pyrano [2,3-d]pyrimidin8-one (2g): Pale yellow solid; IR (KBr) $v_{\max }\left(\mathrm{cm}^{-1}\right)$ : $3281(\mathrm{NH}), 1751$ (CO of lactone), 1737 (CO of lactam), $1601(\mathrm{C}=\mathrm{N}), 1153,1127(\mathrm{C}-\mathrm{O}-\mathrm{C}) ;{ }^{1} \mathrm{H}$ NMR $\left(300 \mathrm{MHz}, \mathrm{DMSO}-d_{6}\right): \delta 3.70(\mathrm{~s}, 6 \mathrm{H}), 4.84(\mathrm{~s}, 1 \mathrm{H})$, $6.72-6.75(\mathrm{~m}, 1 \mathrm{H}), 6.89(\mathrm{~d}, J=6.0 \mathrm{~Hz}, 2 \mathrm{H}), 7.14(\mathrm{~d}$, $J=6.3 \mathrm{~Hz}, 1 \mathrm{H}), 7.39-7.45(\mathrm{~m}, 3 \mathrm{H}), 7.56-7.63(\mathrm{~m}$, $2 \mathrm{H}), 7.71-7.92(\mathrm{~m}, 3 \mathrm{H}), 8.22(\mathrm{~d}, J=6.3 \mathrm{~Hz}, 1 \mathrm{H}), 8.75$ (s, $1 \mathrm{H}), 10.84(\mathrm{~s}, 1 \mathrm{H}) ;{ }^{13} \mathrm{C}$ NMR $\left(75 \mathrm{MHz}, \mathrm{DMSO}-d_{6}\right)$ : 173.0, 164.3, 163.9, 160.0, 156.6, 154.4, 147.7, 142.2, 138.1, 134.2, 132.6, 130.1, 127.6, 126.6, 126.5, 126.2, 124.7, 123.6, 122.7, 120.6, 120.4, 119.8, 118.3, 118.0, 117.9, 116.0, 112.1, 105.9, 55.4, 40.4; MS: $m / z=531$ $(\mathrm{M}+1)$; Anal. Calcd. For $\mathrm{C}_{32} \mathrm{H}_{22} \mathrm{~N}_{2} \mathrm{O}_{6}$ : C, 72.45; $\mathrm{H}$, 4.18; N, 5.28. Found: C, 72.62; H, 4.38; N, 5.03. 2.3h 7-(4-Hydroxyphenyl)-10-(2-oxo-2H-chromen-3-yl)$9 H$-naphtho $\left[3^{\prime}, 4^{\prime}: 5,6\right]$ pyrano $[2,3-d]$ pyrimidin-8-one (2h): Pale yellow solid; IR $(\mathrm{KBr}) v_{\max }\left(\mathrm{cm}^{-1}\right): 3429$ $(\mathrm{OH}), 3294(\mathrm{NH}), 1770$ (CO of lactone), 1719 (CO of lactam), $1603(\mathrm{C}=\mathrm{N}), 1171(\mathrm{C}-\mathrm{O}-\mathrm{C}) ;{ }^{1} \mathrm{H}$ NMR $\left(300 \mathrm{MHz}, \mathrm{DMSO}-d_{6}\right): \delta 4.76(\mathrm{~s}, 1 \mathrm{H}), 6.69(\mathrm{~d}, J=$ $6.3 \mathrm{~Hz}, 2 \mathrm{H}), 7.02-7.10(\mathrm{~m}, 3 \mathrm{H}), 7.39-7.45(\mathrm{~m}, 2 \mathrm{H})$, 7.56-7.64 (m, 3H), 7.71-7.92 (m 3H), $8.22(\mathrm{~d}, J=$ $6.0 \mathrm{~Hz}, 1 \mathrm{H}), 8.75(\mathrm{~s}, 1 \mathrm{H}), 9.30(\mathrm{~s}, 1 \mathrm{H}), 10.75(\mathrm{~s}, 1 \mathrm{H})$; ${ }^{13} \mathrm{C}$ NMR $\left(75 \mathrm{MHz}, \mathrm{DMSO}-d_{6}\right): 173.2,164.3,163.5$, 160.0, 157.1, 154.7, 144.5, 142.8, 134.2, 132.6, 129.8, 128.6, 127.6, 126.8, 126.7, 126.2, 124.9, 123.6, 122.7, 120.5, 120.4, 118.6, 117.9, 117.4, 116.1, 105.9, 38.1; MS: $m / z=487(\mathrm{M}+1)$; Anal. Calcd. For $\mathrm{C}_{30} \mathrm{H}_{18} \mathrm{~N}_{2} \mathrm{O}_{5}$ : C, 74.07; H, 3.73; N, 5.76; Found: C, 74.21; H, 3.48; N, 5.88 .

$2.3 \mathrm{i}$ 7-(4-Hydroxy-3-methoxyphenyl)-10-(2-oxo-2Hchromen-3-yl)-9H-naphtho[3', $\left.4^{\prime}: 5,6\right]$ pyrano[2,3-d]pyrimidin-8-one (2i): Pale yellow solid; IR ( $\mathrm{KBr})$ $v_{\max }\left(\mathrm{cm}^{-1}\right)$ : $3417(\mathrm{OH}), 3280(\mathrm{NH}), 1762(\mathrm{CO}$ of lactone), 1724 (CO of lactam), $1610(\mathrm{C}=\mathrm{N}), 1182,1138$ $(\mathrm{C}-\mathrm{O}-\mathrm{C}) ;{ }^{1} \mathrm{H}$ NMR $\left(300 \mathrm{MHz}, \mathrm{DMSO}-d_{6}\right): \delta 3.72(\mathrm{~s}$, $3 \mathrm{H}), 4.78(\mathrm{~s}, 1 \mathrm{H}), 6.60(\mathrm{~d}, J=5.7 \mathrm{~Hz}, 1 \mathrm{H}), 6.70(\mathrm{~d}$, $J=6.3 \mathrm{~Hz}, 1 \mathrm{H}), 6.85(\mathrm{~s}, 1 \mathrm{H}), 7.14(\mathrm{~d}, J=6.3 \mathrm{~Hz}$, 1H), 7.39-7.45 (m, 3H), 7.56-7.62 (m, 2H), 7.71-7.92 $(\mathrm{m}, 3 \mathrm{H}), 8.22(\mathrm{~d}, J=6.0 \mathrm{~Hz}, 1 \mathrm{H}), 8.75(\mathrm{~s}, 1 \mathrm{H}), 8.88$ $(\mathrm{s}, 1 \mathrm{H}), 10.64(\mathrm{~s}, 1 \mathrm{H}) ;{ }^{13} \mathrm{C}$ NMR $\left(75 \mathrm{MHz}, \mathrm{DMSO}-d_{6}\right)$ : 173.6, 164.4, 163.9, 159.9, 156.6, 154.4, 145.5, 142.4, $136.6,134.2,132.5,130.1,127.6,126.5,126.2,124.7$, 123.6, 122.7, 120.6, 120.0, 118.3, 117.9, 116.0, 115.6, 105.9, 55.6, 40.4; MS: $m / z=517(\mathrm{M}+1)$; Anal. Calcd. For $\mathrm{C}_{31} \mathrm{H}_{20} \mathrm{~N}_{2} \mathrm{O}_{6}$ : C, 72.09; H, 3.90; N, 5.42; Found: C, $72.26 ; \mathrm{H}, 3.74 ; \mathrm{N}, 5.62$.

2.3j 7-(4-Dimethylamino-phenyl)-10-(2-oxo- $2 \mathrm{H}$ chromen-3-yl)-9H-naphtho[3',4':5,6] pyrano[2,3-d]pyrimidin-8-one $(\mathbf{2 j})$ : Colourless solid; IR $(\mathrm{KBr}) v_{\max }$ $\left(\mathrm{cm}^{-1}\right): 3274(\mathrm{NH}), 1767$ (CO of lactone), 1725 (CO of lactam), $1605(\mathrm{C}=\mathrm{N}), 1182(\mathrm{C}-\mathrm{O}-\mathrm{C}) ;{ }^{1} \mathrm{H}$ NMR $\left(300 \mathrm{MHz}, \mathrm{DMSO}-d_{6}\right): \delta 3.11(\mathrm{~s}, 6 \mathrm{H}), 4.93(\mathrm{~s}, 1 \mathrm{H})$, $6.85(\mathrm{~d}, J=6.6 \mathrm{~Hz}, 2 \mathrm{H}), 7.39-7.45(\mathrm{~m}, 5 \mathrm{H}), 7.71-7.75$ $(\mathrm{m}, 3 \mathrm{H}), 7.83(\mathrm{~d}, J=6.9 \mathrm{~Hz}, 2 \mathrm{H}), 7.91(\mathrm{~d}, J=5.7 \mathrm{~Hz}$, 2H), 8.75 (s, 1H), 10.92 (s, 1H); ${ }^{13} \mathrm{C}$ NMR $(75 \mathrm{MHz}$, DMSO- $\left.d_{6}\right)$ : $173.6,164.4,163.5,160.1,154.7,148.2$, 144.6, 142.3, 132.9, 130.1, 129.7, 128.7, 127.6, 126.8, $126.5,126.1,124.9,123.8,122.7,120.7,120.3,118.3$, 117.9, 117.3, 115.3, 106.7, 40.7, 38.0; MS: $m / z=514$ $(\mathrm{M}+1)$; Anal. Calcd. For $\mathrm{C}_{32} \mathrm{H}_{23} \mathrm{~N}_{3} \mathrm{O}_{4}$ : C, 74.84; $\mathrm{H}$, 4.51 ; N, 8.18; Found: C, 74.66; H, 4.72; N, 8.04. 


\section{Results and discussions}

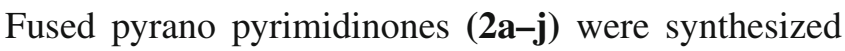
by the condensation of 2-amino-4-aryl- $4 \mathrm{H}$-benzo$[h]$ chromene-3-carbonitriles (1a-j) with 2-oxo- $2 \mathrm{H}$ chromene-3-carboxylic acid under solvent-free conditions at $120^{\circ} \mathrm{C}$ utilizing Brønsted acidic ionic liquid, (4-sulfobutyl)tris(4-sulfophenyl)phosphonium hydrogen sulphate as green catalyst (scheme 1). The ionic liquid (IL) has been prepared according to the literature procedure. ${ }^{33}$

Reaction between 2-amino-4-phenyl-4H-benzo[ $h]$ chromene-3-carbonitrile (1a) and 2-oxo-2 $\mathrm{H}$-chromene3 -carboxylic acid was carried out as a modal reaction at different temperatures (Rt, 80, 120 and $150^{\circ} \mathrm{C}$ ) by varying the amount of ionic liquid (5, 10 and $15 \mathrm{~mol} \%)$ under solvent-free conditions. The maximum yield of the product $2 \mathbf{a}$ was observed at $120^{\circ} \mathrm{C}$ with $10 \mathrm{~mol} \%$ of ionic liquid. The efficiency of the ionic liquid was also compared with different acid catalysts (sulphuric acid, silica sulphuric acid and cellulose sulphuric acid) and the results were presented in table 1.

At these optimizing conditions (solvent-free, $120^{\circ} \mathrm{C}$, $10 \mathrm{~mol} \%$ of IL), we performed the reaction using different 2-amino-4-aryl-4H-benzo[ $h]$ chromene-3-carbonitrile $(\mathbf{1 a}-\mathbf{j})$ and synthesized the titled compounds $(\mathbf{2} \mathbf{a}-\mathbf{j})$ in good yields (table 2). After completion of the reaction indicated by TLC, the catalyst was recovered by evaporating the aqueous layer, washed with acetone, dried and reused for subsequent reactions without significant loss in its activity and product yield. For example, the reaction of 2-amino-4-phenyl- $4 H$-benzo[ $h]$ chromene-

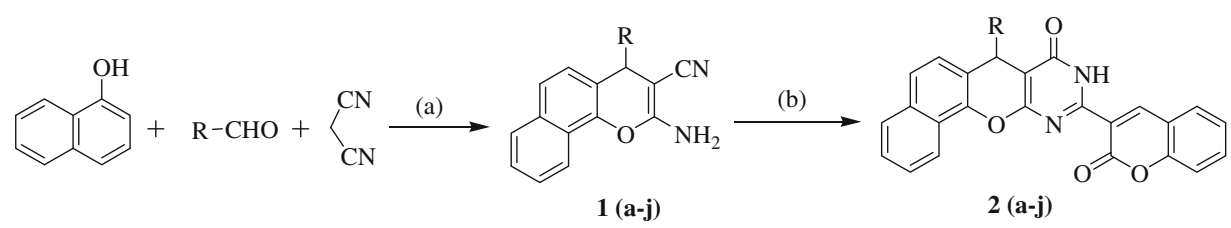

\begin{tabular}{c|l} 
Compound & $\mathbf{R}$ \\
\hline $\mathbf{1 a} / \mathbf{2 a}$ & $\mathrm{C}_{6} \mathrm{H}_{5}$ \\
$\mathbf{1 b} / \mathbf{2 b}$ & $2-\mathrm{ClC}_{6} \mathrm{H}_{4}$ \\
$\mathbf{1 c} / \mathbf{2 c}$ & $4-\mathrm{ClC}_{6} \mathrm{H}_{4}$ \\
$\mathbf{1 d} / \mathbf{2 d}$ & $3-\mathrm{NO}_{2} \mathrm{C}_{6} \mathrm{H}_{4}$ \\
$\mathbf{1 e} / \mathbf{2} \mathbf{e}$ & $4-\mathrm{CH}_{3} \mathrm{C}_{6} \mathrm{H}_{4}$ \\
$\mathbf{1 f} / \mathbf{2 f}$ & $4-\mathrm{OCH}_{3} \mathrm{C}_{6} \mathrm{H}_{4}$ \\
$\mathbf{1 g} / \mathbf{2} \mathbf{g}$ & $3,4-\left(\mathrm{OCH}_{3}\right)_{2} \mathrm{C}_{6} \mathrm{H}_{3}$ \\
$\mathbf{1 h} / \mathbf{2} \mathbf{h}$ & $4-\mathrm{OHC} \mathrm{H}_{4}$ \\
$\mathbf{1 i} / \mathbf{2} \mathbf{i}$ & $4-\mathrm{OH}-3-\mathrm{OCH}_{3} \mathrm{C}_{6} \mathrm{H}_{3}$ \\
$\mathbf{1 j} / \mathbf{2} \mathbf{j}$ & $4-\mathrm{N}\left(\mathrm{CH}_{3}\right)_{2} \mathrm{C}_{6} \mathrm{H}_{4}$
\end{tabular}

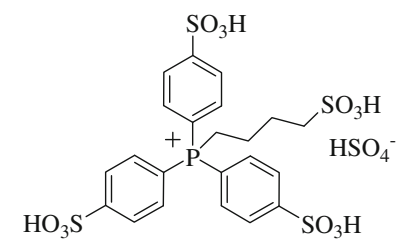

Ionic liquid, (4-sulfobutyl)tris(4-sulfophenyl) phosphonium hydrogen sulphate (IL)

Reaction conditions: (a) Ethanol, $\mathrm{Et}_{3} \mathrm{~N}$, Reflux, 1-6 h; (b) 2-oxo-2H-chromene-3-carboxylic acid, Ionic liquid (10 mol\%), Neat, $120^{\circ} \mathrm{C}, 8-12 \mathrm{~h}$.

Scheme 1. Synthetic pathway of the fused pyrano pyrimidinones.

Table 1. Optimization of the reaction conditions. ${ }^{\text {a }}$

\begin{tabular}{llccc}
\hline S.No. & \multicolumn{1}{c}{ Catalyst (mol\%) } & Temp. $\left({ }^{\circ} \mathrm{C}\right)$ & Time $(\mathrm{h})$ & Yield $(\%)$ \\
\hline 1 & Ionic liquid (5 mol\%) & RT & 24 & - \\
2 & Ionic liquid (5 mol\%) & 80 & 12 & 28 \\
3 & Ionic liquid (5 mol\%) & 120 & 12 & 36 \\
4 & Ionic liquid (5 mol\%) & 150 & 12 & 37 \\
5 & Ionic liquid (10 mol\%) & 80 & 12 & 57 \\
6 & Ionic liquid (10 mol\%) & 120 & 10 & 72 \\
7 & Ionic liquid (10 mol\%) & 150 & 10 & 72 \\
8 & Ionic liquid (15 mol\%) & 120 & 10 & 72 \\
9 & Ionic liquid (15 mol\%) & 150 & 10 & 72 \\
10 & Sulphuric acid (10 mol\%) & 120 & 10 & 19 \\
11 & Silica sulphuric acid (10 mol\%) & 120 & 10 & 36 \\
12 & Cellulose sulphuric acid (10 mol\%) & 120 & 10 & 48 \\
\hline
\end{tabular}

a Reaction conditions: 2-amino-4-phenyl-4H-benzo[ $h]$ chromene-3-carbonitrile ( $1 \mathrm{mmol}$ ), 2-oxo-2 $\mathrm{H}$-chromene-3-carboxylic acid ( $1 \mathrm{mmol})$, solvent-free conditions.

${ }^{\mathrm{b}}$ Yields refer to isolated pure product (2a) 
3-carbonitrile (1a) with 2-oxo-2H-chromene-3carboxylic acid gave the corresponding fused pyrano pyrimidinone (2a) in 72, 70, 70, 69 and 68\% yields over additional five cycles. All the compounds were characterized by IR, NMR and Mass spectral data.

\section{Biological activities}

All the synthesized compounds $(\mathbf{2} \mathbf{a}-\mathbf{j})$ were screened for in vitro antibacterial activity against Gram-positive bacterial strains: Bacillus subtilis (Bs), Staphylococcus aureus (Sa) and Staphylococcus epidermidis (Se), and Gram-negative bacterial strains: Escherichia coli $(\mathrm{Ec})$,
Pseudomonas aeruginosa $(\mathrm{Pa})$ and Klebsiella pneumonia $(\mathrm{Kp})$ with respect to Penicillin and Streptomycin as the positive control drugs. Antifungal screening was carried out against Aspergillus flavus (Af), Saccharomyces cerevisiae $(\mathrm{Sc})$, Candida rugosa $(\mathrm{Cr})$ and Candida albicans $(\mathrm{Ca})$ fungal strains using Amphotericin-B as the positive control drug.

\subsection{Antibacterial activity}

The minimum inhibitory concentration (MIC) values for analogs and the positive control drugs Penicillin and Streptomycin were determined against the six

Table 2. Synthesis of fused pyrano pyrimidinones $2(\mathbf{a}-\mathbf{j})$ using ionic liquid (IL). ${ }^{\mathrm{a}}$

\begin{tabular}{|c|c|c|c|c|}
\hline Analog & $\mathrm{R}$ & Time (h) & Yield $^{\mathrm{b}}(\%)$ & M.p $\left({ }^{\circ} \mathrm{C}\right)$ \\
\hline $2 \mathbf{a}$ & $\mathrm{C}_{6} \mathrm{H}_{5}$ & 10 & 72 & $277-279$ \\
\hline $2 \mathbf{b}$ & $2-\mathrm{ClC}_{6} \mathrm{H}_{4}$ & 12 & 68 & $290-292$ \\
\hline $2 c$ & $4-\mathrm{ClC}_{6} \mathrm{H}_{4}$ & 8 & 76 & $222-224$ \\
\hline $2 d$ & $3-\mathrm{NO}_{2} \mathrm{C}_{6} \mathrm{H}_{4}$ & 12 & 71 & $231-233$ \\
\hline $2 e$ & $4-\mathrm{CH}_{3} \mathrm{C}_{6} \mathrm{H}_{4}$ & 9 & 67 & $244-246$ \\
\hline $2 f$ & $4-\mathrm{OCH}_{3} \mathrm{C}_{6} \mathrm{H}_{4}$ & 10 & 73 & $267-270$ \\
\hline $2 \mathrm{~g}$ & $3,4-\left(\mathrm{OCH}_{3}\right)_{2} \mathrm{C}_{6} \mathrm{H}_{3}$ & 10 & 74 & $255-257$ \\
\hline $2 \mathrm{~h}$ & $4-\mathrm{OHC}_{6} \mathrm{H}_{4}$ & 12 & 69 & $242-244$ \\
\hline $2 \mathbf{i}$ & $4-\mathrm{OH}-3-\mathrm{OCH}_{3} \mathrm{C}_{6} \mathrm{H}_{3}$ & 8 & 77 & 249-251 \\
\hline $2 \mathbf{j}$ & $4-\mathrm{N}\left(\mathrm{CH}_{3}\right)_{2} \mathrm{C}_{6} \mathrm{H}_{4}$ & 12 & 72 & $270-272$ \\
\hline
\end{tabular}

Table 3. Minimum inhibitory concentration (MIC) values for fused pyrano pyrimidinones (2a-j) and positive control drugs against different bacterial strains.

\begin{tabular}{|c|c|c|c|c|c|c|}
\hline \multirow[b]{3}{*}{ Analog } & \multicolumn{6}{|c|}{ Minimum inhibitory concentration $(\mu \mathrm{g} / \mathrm{mL})$} \\
\hline & \multicolumn{3}{|c|}{ Gram-positive bacteria } & \multicolumn{3}{|c|}{ Gram-negative bacteria } \\
\hline & Bs & $\mathrm{Sa}$ & $\mathrm{Se}$ & Ec & $\mathrm{Pa}$ & $\mathrm{Kp}$ \\
\hline $2 \mathbf{a}$ & 150 & 300 & 150 & 75 & 150 & 150 \\
\hline $2 b$ & 150 & 300 & 150 & 75 & 150 & 300 \\
\hline $2 \mathrm{c}$ & 150 & 150 & 150 & 37.5 & 150 & 150 \\
\hline 2d & 150 & 300 & 150 & 37.5 & 150 & 150 \\
\hline $2 \mathrm{e}$ & 150 & 300 & 150 & 75 & 150 & 150 \\
\hline $2 f$ & 300 & 450 & 300 & 75 & 150 & 150 \\
\hline $2 \mathrm{~g}$ & 150 & 300 & 300 & 75 & 150 & 150 \\
\hline $2 \mathrm{~h}$ & 450 & 300 & 150 & 75 & 150 & 300 \\
\hline $2 \mathbf{i}$ & 450 & 450 & 150 & 75 & 150 & 150 \\
\hline $2 \mathbf{j}$ & 150 & 150 & 150 & 75 & 150 & 150 \\
\hline Penicillin & 1.562 & 1.562 & 3.125 & 12.5 & 12.5 & 6.25 \\
\hline Streptomycin & 6.25 & 6.25 & 3.125 & 6.25 & 1.562 & 3.125 \\
\hline
\end{tabular}

Gram-positive bacteria: Bs: Bacillus subtilis; Sa: Staphylococcus aureus; Se: Staphylococcus epidermidis.

Gram-negative bacteria: Ec: Escherichia coli; Pa: Pseudomonas aeruginosa; Kp:

Klebsiella pneumonia 
Table 4. Zone of inhibition data for fused pyrano pyrimidinones 2(a-j) against different fungal strains at 100 and $150 \mu \mathrm{g} / \mathrm{mL}$ concentrations.

\begin{tabular}{|c|c|c|c|c|c|c|c|c|}
\hline \multirow[b]{3}{*}{ Analog } & \multicolumn{8}{|c|}{ Zone of inhibition (mm) } \\
\hline & \multicolumn{2}{|c|}{ Af } & \multicolumn{2}{|c|}{$\mathrm{Sc}$} & \multicolumn{2}{|c|}{$\mathrm{Cr}$} & \multicolumn{2}{|c|}{$\mathrm{Ca}$} \\
\hline & $100 \mu \mathrm{g}$ & $150 \mu \mathrm{g}$ & $100 \mu \mathrm{g}$ & $150 \mu \mathrm{g}$ & $100 \mu \mathrm{g}$ & $150 \mu \mathrm{g}$ & $100 \mu \mathrm{g}$ & $150 \mu \mathrm{g}$ \\
\hline $2 \mathbf{a}$ & 11 & 14 & - & - & - & - & 14 & 18 \\
\hline $2 b$ & 12 & 16 & - & - & 11 & 15 & - & - \\
\hline $2 c$ & 11 & 15 & - & - & 09 & 14 & - & - \\
\hline $2 d$ & 09 & 12 & - & - & - & - & 09 & 14 \\
\hline $2 e$ & 08 & 11 & - & - & 11 & 16 & - & - \\
\hline $2 f$ & - & - & - & - & 16 & 20 & 12 & 16 \\
\hline $2 g$ & 15 & 21 & - & - & 18 & 22 & 14 & 18 \\
\hline $2 \mathrm{~h}$ & - & - & - & - & 11 & 15 & 12 & 15 \\
\hline $2 \mathbf{i}$ & 7 & 11 & - & - & 12 & 18 & - & - \\
\hline & 13 & 16 & - & - & 12 & 16 & 11 & 15 \\
\hline AMP-B & 2 & & 2 & & 2 & & 23. & \\
\hline
\end{tabular}

Fungal strains: Af: Aspergillus flavus; Sc: Saccharomyces cerevisiae; Cr: Candida rugosa; $\mathrm{Ca}$ : Candida albicans.

AMP-B: Amphotericin-B; '-' not active

bacterial strains by the liquid dilution method. ${ }^{34,35}$ Different concentrations of analogs and positive control drugs were prepared in DMSO. Inoculums of the bacterial cultures were also prepared. Inoculum $(0.2 \mathrm{~mL})$ and sterile water $(3.8 \mathrm{~mL})$ were added to a series of test tubes each containing $1 \mathrm{~mL}$ of test solution at different concentrations.

The tubes were incubated for $24 \mathrm{~h}$ at $37^{\circ} \mathrm{C}$ and carefully observed for the presence of turbidity. The minimum concentration at which no growth was observed was taken as the MIC value (table 3 ).

From the activity report (table 3 ) it was notified that, the compounds $\mathbf{2 c}$ and $\mathbf{2 d}$ have shown good activity against Escherichia coli (Gram-negative bacteria) with MIC $37.5 \mu \mathrm{g} / \mathrm{mL}$ compare to the standard drug Penicillin, remaining all the compounds have shown moderate activity against all the tested bacterial strains.

\subsection{Antifungal activity}

Zone of inhibition (in $\mathrm{mm}$ ) values for analogs and positive control drug Ampothericin-B were determined against four different fungal strains by Agar discdiffusion method. ${ }^{36,37}$ All the compounds were dissolved in DMSO. The fungal strains were grown and maintained on Sabouraud glucose agar plates. The plates were incubated at $26^{\circ} \mathrm{C}$ for $48 \mathrm{~h}$, and resulting ZOIs were measured. Antifungal screening for analogs and positive control was performed at a concentration of 100 and $150 \mu \mathrm{g} / \mathrm{mL}$ and the results are illustrated in table 4.

The zone of inhibition data indicating that the compound $\mathbf{2 g}$ has shown good activity against Candida rugosa $(\mathrm{ZOI}=22 \mathrm{~mm})$ at $150 \mu \mathrm{g} / \mathrm{mL}$ when compared to the standard drug Amphotericin-B, remaining compounds have shown moderate activity and all the compounds were inactive against Saccharomyces cerevisiae.

\section{Conclusion}

Various fused pyrano pyrimidinones $(\mathbf{2 a - j})$ were synthesized using an efficient, eco-friendly and reusable Brønsted acidic ionic liquid, (4-sulfobutyl)tris(4sulfophenyl)phosphonium hydrogen sulphate as catalyst under neat conditions. All the synthesized compounds were screened for in vitro antibacterial and antifungal activity against different bacterial and fungal strains. The antimicrobial screening data revealed that the compounds $\mathbf{2 c}$ and $\mathbf{2 d}$ have shown good activity against Gram-negative bacteria Escherichia coli, where as compound $\mathbf{2 g}$ has shown superior antifungal activity against Candida rugosa at $150 \mu \mathrm{g} / \mathrm{mL}$ compare to the standard antifungal drug Amphotericin-B.

\section{Acknowledgements}

The authors thank the Department of Biotechnology, Kakatiya University, India for screening the antimicrobial activity of the synthesized compounds. B J thanks Ministry of Human Resources Department (MHRD) and R G and R V thank the University Grants Commission (UGC) for providing research fellowship. 


\section{References}

1. Zhang G, Zhang Y, Yan J, Chen R, Wang S, Ma Y and Wang R 2012 J. Org. Chem. 77878

2. Chetan B S, Divyesh C M, Manish P P and Ranjan G P 2012 Chin. Chem. Lett. 2357

3. E1-Gaby M S A, Zahran M A, Ismail M M F and Ammar Y A 2000 Il Farmaco 55227

4. Divyesh C M, Manish P P, Dhanji P R and Ranjan G P 2011 Eur. J. Med. Chem. 464192

5. Hwan $\mathrm{K} J$, Eun $\mathrm{K} \mathrm{H}$, Kyung J J, Hwajung $\mathrm{K}$, Jungsook C and Heesoon L 2006 Arch. Pharm. Res. 29 728

6. Fakher C, Mehdi M, Hedi B M, Leila C and Mansour S 2007 Eur. J. Med. Chem. 42715

7. Ren S C, Sheau F Y, Chih M L, Amooru G, Damu T K, Cheng C P, Kenneth F B, Hsiung L K and Shung W T 2009 Bioorg. Med. Chem. 176137

8. Cheng C Y, Chiu H, Chang M J, Lin Y C, Tsai M C and Yu H C 1998 Bioorg. Med. Chem. Lett. 8463

9. Abdou O A, Ahmed H E, Sayed A A and Yasser H Z 2005 J. Sulf. Chem. 26405

10. Modrana J N, Nawrot E and Graczyk J 2006 Eur. J. Med. Chem. 411301

11. Sardari S, Mori Y, Horita K, Micetich R G, Nishibe S and Daneshtalab M 1999 Bioorg. Med. Chem. 7 1933

12. Nimesh R K, Dhaval D H, Prashant T M and Saurabh K P 2010 Eur. J. Med. Chem. 455056

13. Rai U S, Arun M I, Prakash S, Vijesh A M, Nithin P, Shrikrishna I, Thiageeswaran M and Hoong-Kun F 2010 Eur. J. Med. Chem. 452695

14. Cecile G, Douguet D, Huteau V, Gilles M, Helene M L and Sylvie P 2008 Bioorg. Med. Chem. 166075

15. Massimo C, Francesco E, Federica M, Carla M M, Prieto G S and Carlos R J 2003 Aust. J. Chem. 5659

16. Bhat M A, Siddiqui N and Khan S A 2006 Indian J. Pharm. Sci. 68120

17. Ruszat R, Wyler S, Forster T, Reich O, Christian G S, Thomas C G, Sulser T and Bachmann A 2006 Eur. Assoc. Urol. $\mathbf{5 0} 675$
18. Tyagi A K, Raj H G, Vohra P, Gupta G, Kumari R, Kumar P and Gupta R K 2003 Eur. J. Med. Chem. 40413

19. Huang L, Yuon X, Yu D, Lee K H and Chin H C 2005 Virology 332623

20. Mohanty N, Rath P C and Rout M K 1967 J. Indian Chem. Soc. 441001

21. Deshmukh M B, Salunkhe S M, Patil D R and Anbhule P V 2009 Eur. J. Med. Chem. 442651

22. Lin R, Sigmond G, Johnson P J, Connolly S K, Wetter E, Binnun T V, Hughes W V, Murray N B, Pandey S J, Mazza M M, Adams A R, Pesquera F and Steven A M 2009 Bioorg. Med. Chem. Lett. 192333

23. Lin C M, Huang S T, Lee F W, Sawkuo H and Lin M H 2006 Bioorg. Med. Chem. 144402

24. Falcao E P, Da S, Melo S J, Srivastava R M, Catanho M T and Nascimento S C 2006 Eur. J. Med. Chem. 41 276

25. Agarwal A, Ashutosh R, Goyal N, Chauhana M S and Gupta S 2005 Bioorg. Med. Chem. 136678

26. Shu-Liang W, Ke Y and Xiang-Shan W 2011 Chin. J. Org. Chem. 311235

27. Jing X, Lib Z, Wu L and Yan C 2011 J. Iran. Chem. Soc. 8107

28. Moni S, Shashi P, Kuldeep C, Deepty S, Brijesh K and Prem M S C 2012 J. Org. Chem. 77929

29. Heravi M M, Tavakoli-Hoseini N and Bamoharram F 2011 Synth. Commun. 41707

30. Martyn J E and Kenneth R S 2000 Pure Appl. Chem. 72 1391

31. Sheldon R A 1996 J. Mol. Catal. A: Chem. 10775

32. Janardhan B and Rajitha B 2012 Chin. Chem. Lett. 23 1015

33. Hamid R S, Mohammad R and Kobra A 2011 J. Mol. Liq. 16295

34. Omrum U, Arikan S, Kocago S, Semeak B and Unala D 2000 Microbiol. Infect. Dis. 38101

35. Malue M, Bastide J M and Biancard A 2005 Int. J. Antimicrob. Agents 25321

36. Alam S 2004 Chem. Sci. 166325

37. Reddy P M, Ho Y P, Shanker K, Rohini R and Ravinder V 2009 Eur. J. Med. Chem. 442621 